Diabetologia $9,235-238(1973)$

(C) by Springer-Verlag 1973

\title{
Diurnal Variation in Oral Glucose Tolerance: Insulin and Growth Hormone Changes with Special Reference to Women Taking Oral Contraceptives
}

\author{
N.W. Oakley, D. Monier and V. Wynn \\ Alexander Simpson Laboratory for Metabolic Research, St. Mary's Hospital Medical Sehool, London, W. 2
}

Received: November 8, 1972 and in revised form January 29, 1973, accepted: February 9,1973

Summary. Plasma glucose, immunoreactive insulin, and growth hormone (GH) have been estimated during morning (AM) and afternoon (PM) oral glucose tolerance tests (GTT) in a group of 22 subjects, 13 of whom were young women receiving combined oestrogen-progestogen oral contraceptives. Impaired PM glucose tolerance with associated delay and impairment of insulin secretion has been confirmed, as has the inverse correlation between obesity and diurnal GTT variation. Diumal changes in GH are unlikely to be responsible for this circadian GTT rhythm. Patients on oral contraceptives continue to show a normal GTT rhythm, so that a mildly abnormal AM test is likely to be associated with a more severely diabetic PM test. These observations indicate that while obesity and oral contraceptive use may have metabolic features in common, they differ in this respect; they support the view that, if possible, carbohydrate tolerance should be monitored in women taking oral contraceptives in whom a tendency to diabetes is suspected.

Key words: Diurnal rhythm, glucose tolerance, insulin, growth hormone, oral contraceptives, obesity.

\section{Introduction}

It is believed that about $15 \%$ of otherwise normal women develop chemical diabetes when taking combined oestrogen-progestogen oral contraceptives [1]. It has also been suggested that many of the metabolic features of patients receiving these drugs are similar to those found in obesity - a situation perhaps explicable on the basis of increased glucocorticoid activity in both instances [2].

One feature of obesity which has so far not been reported in patients receiving oral contraceptives is the loss of diurnal glucose tolerance rhythm described in overweight subjects [3]. Glucose tolerance tests in women on oral contraceptives have always been carried out in the morning; there is therefore no information as to whether, as in obese subjects, an afternoon test would give similar results to a morning one or whether, as in normal subjects, deterioration would occur during the day. If the former situation were found to obtain, it could be supposed that carbohydrate tolerance would only be impaired for a small part of the day in women on the "pill" - a state of affairs which could perhaps be regarded as less potentially serious than one in which mild carbohydrate intolerance in the morning was associated with a high incidence of frank chemical diabetes later in the day.

Previous work on the subject of diurnal variation in glucose tolerance has largely been carried out on men [4, 3]. A group of 22 subjects, including 13 women on combined oral contraceptive preparations has now been studied. The opportunity was taken to estimate plasma insulin and growth hormone (GH) during the tests, and again to examine the effect of obesity on the diurnal glucose tolerance rhythm.

\section{Material and Methods}

Oral glucose tolerance tests $(1 \mathrm{~g} / \mathrm{kg}$, in $360 \mathrm{ml}$ water) were carried out on 22 subjects, 21 women and $1 \mathrm{man}, 13$ of the women being on combined oestrogenprogestogen oral contraceptive preparations. Each subject received a diet containing at least $200 \mathrm{~g}$ carbohydrate for 3 days before each test; the tests were all carried out with the subject semi-recumbent in bed, having rested for at least $30 \mathrm{~min}$ to establish basal conditions after insertion of a polythene canula ("Braunula") for blood sampling.

Tests were carried out under two conditions:

A.M. at 9.00 a.m. after a fast from 10.00 p.m. the previous night

P.M. at 3.00 p.m. after having only had a light dry breakfast on the morning of the test.

This arrangement was as close to comparable conditions of preparation as could be obtained in out-patients. It was regarded as desirable to perform the tests on outpatients rather than in-patients, to sample a situation as close as possible to the normal pattern of daily life.

Blood samples were taken into lithium heparin tubes (Stayne laboratories) and kept in ice for up to $2 \mathrm{~h}$ prior to separation of plasma for analysis. Glucose was estimated for all tests, using the autoanalyser glucose oxidase method of Cramp [5]. Insulin and growth hormone were estimated on samples from 17 pairs of tests, using a combined radio-immunoassay technique with 125-I GH and 131-I insulin, based on published methods $[6,7]$.

\section{Results}

\section{A) Glucose}

Plasma glucose has been estimated on the whole series of patients, as shown on the left of Fig. 1. The 
afternoon pattern is clearly different from that found in the morning, significance of $p<0.05$ or better being obtained (paired Student's t-test) from 60-150 min after glucose. There is only a small difference between
The pattern of AM and PM tests is very similar to that seen for the group as a whole, and there is no evidence that the impaired carbohydrate tolerance of women on $\mathrm{O} / \mathrm{C}$ is due to loss of diurnal rhythm.

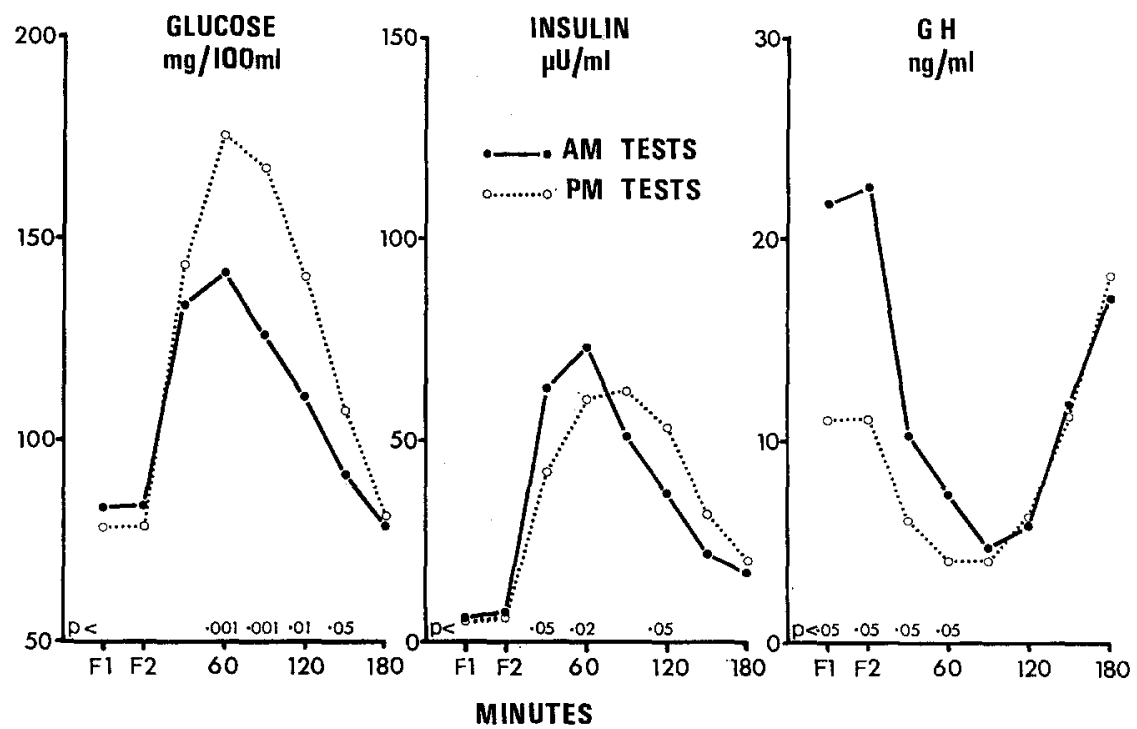

Fig. 1. Mean plasma glucose, insulin and growth hormone values before (F1 and F2) and after (time in minutes) oral glucose $1 \mathrm{~g} / \mathrm{kg}$ for all subjects tested. Solid lines represent morning (AM) tests, dotted lines afternoon (PM) tests. Values for $\mathrm{p}$ are calculated using paired Student's t-test

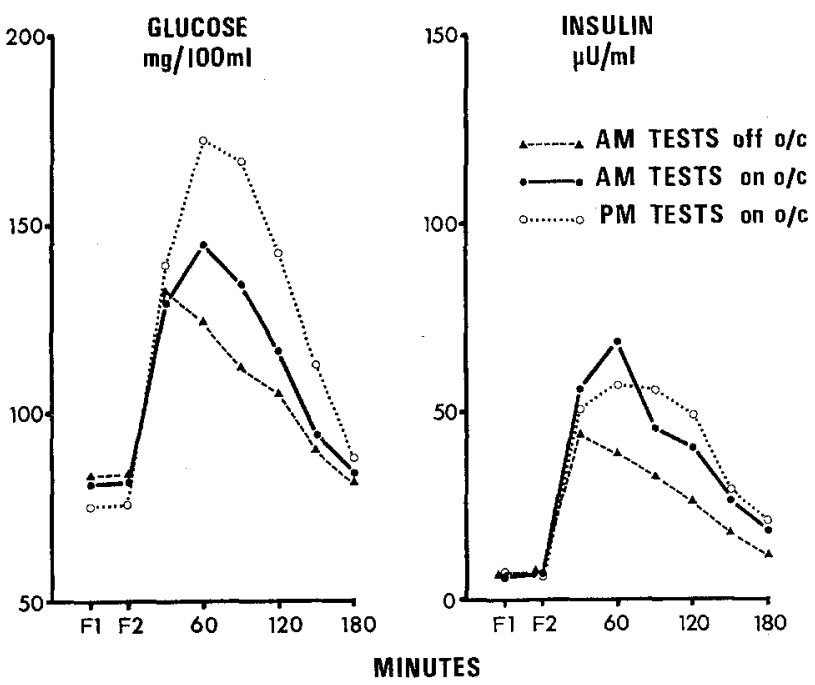

Fig. 2. Mean plasma glucose and insulin values before (F1 and F2) and after (time in minutes) oral glucose $1 \mathrm{~g} / \mathrm{kg}$ for women taking oral contraceptives $(\mathrm{O} / \mathrm{C})$. This figure includes comparable data from women tested in the morning before starting $\mathrm{O} / \mathrm{C}$ (see text)

the fasting values for the AM and PM tests and both have returned to similar values by $180 \mathrm{~min}$.

Glucose values for the oral contraceptive patients $(\mathrm{O} / \mathrm{C})$ are shown on the left side of Fig. 2, which also shows the mean glucose values for 108 comparable pre-O/C women tested in exactly the same way, using the same glucose method in the same laboratory [2].

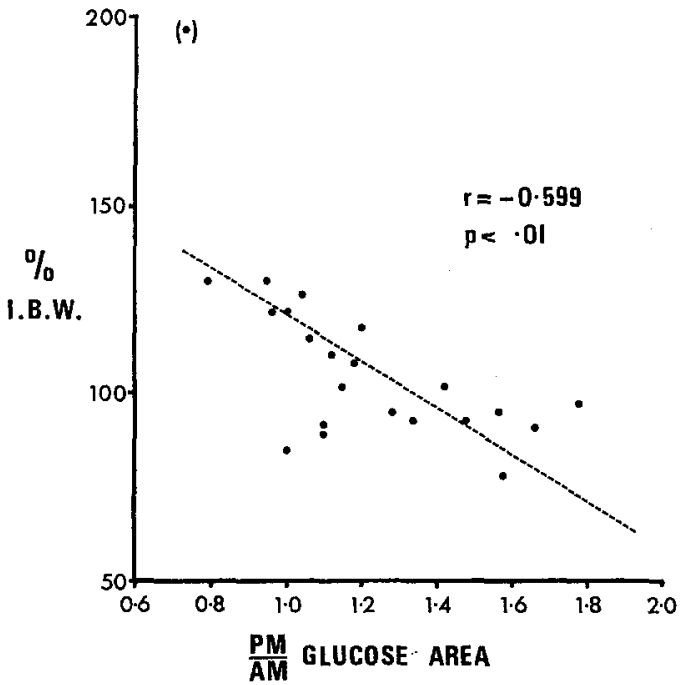

Fig. 3. Correlation between percentage ideal body weight and ratio of areas under PM and AM $3 \mathrm{~h}$ oral glucose tolerance curves, for all patients studied. The value in brackets has not been used in the statistical calculation

In Fig. 3 the ratio of areas under the $3 \mathrm{~h}$ glucose curves for the AM and PM tests has been plotted against the percentage ideal body weight [8]. Even omitting one grossly obese subject, there is a highly significant inverse correlation between these two parameters $(r=-0.599, p<0.01)$ as previously reported [3]. 


\section{B) Insulin}

In the centre of Fig. 1 the mean insulin data is shown for the 17 pairs of tests in which this hormone was estimated. The mean values have been calculated from log-transformed data, to conform with the reported distribution of fasting and post-glucose insulin values $[9,10]$. The most characteristic feature of the PM tests was the delayed rise, with a peak value at $90 \mathrm{~min}$ as compared with $60 \mathrm{~min}$ for the AM tests. From 90 to $180 \mathrm{~min}$ the PM values were higher than the AM ones, the pattern being strongly reminiscent of that seen in AM tests on mild diabetics. Differences between $A M$ and PM mean values reached statistical significance $(p<0.05$ or better) at 30,60 and $120 \mathrm{~min}$, as shown in Fig. 1. The right hand graph of Fig. 2 shows that the pattern was very similar for the patients receiving $\mathrm{O} / \mathrm{C}$ (10 subjects) supporting the impression that morning carbohydrate intolerance on $\mathrm{O} / \mathrm{C}$ was not due to loss of diurnal rhythm. Fig. 2 also shows the insulin data from 38 pre $0 / C$ women, of similar age and weight distribution, whose plasma insulin levels have recently been estimated in our laboratory. The pre $0 / \mathrm{C}$ insulin pattern corresponds closely to the pre o/C glucose pattern, with a peak mean value at $30 \mathrm{~min}$.

\section{C) Growth Hormone}

The right hand graph of Fig. 1 shows mean GH data for the 17 patients on whose samples assays were performed. The AM GH levels were significantly higher than the PM ones up to 60 min after glucose. Nevertheless, suppression of GH by glucose was similar in the two tests. From 90 to $180 \mathrm{~min}, \mathrm{AM}$ and PM GH values were identical. GH values have not been plotted separately for the $\mathrm{O} / \mathrm{C}$ patients only, as they yielded no further information, having exactly the same distribution pattern as the larger group.

\section{Discussion}

The oral glucose tolerance of normal subjects is known to deteriorate during the course of the day [11, 12]. Jarrett and Keen [4] reported loss of diurnal variation in mild diabetes and later demonstrated an inverse relationship between diurnal variation and degree of obesity [3]. The studies described in this report were designed to shed light on the problem of chemical diabetes induced by oral contraceptives, and also to provide further information about the diurnal glucose tolerance rhythm. Statistically significant results were quickly obtained using a small series of patients.

Previous studies revealed that impaired glucose tolerance in the afternoon is associated with delayed and impoverished insulin secretion [13]. The insulin assays carried out for that report were performed in the same laboratory as the present study, but presented greater technical problems, being carried out on capillary plasma, using the method previously developed for very small fetal samples; for the present study, venous plasma samples stored under optimal conditions were used. It was therefore encouraging to obtain exactly the same pattern of insulin secretion in the present study, which yielded AM peak values whose arithmetical means were similar to those previously reported from our laboratory, although fasting values are now lower; the latter were almost certainly due to technical improvements in the assay system (see Fig. 2) and conformed more closely to our own recent results obtained on fasting plasma using a specially sensitive assay technique [9].

The use of oral contraceptives is associated with a rise in both glucose and insulin values (Fig. 2) suggesting that reduced net glucose assimilation, perhaps associated with insulin resistance, is likely to be the primary abnormality. In contrast, afternoon glucose tolerance tests show increased glucose values, with delayed insulin secretion, similar to that seen in the morning in mild diabetes; the major abnormality in both instances appears to be loss of the prompt release of insulin in response to a glucose stimulus.

An endogenous clinical glucose tolerance rhythm could be mediated either through an autonomic pathway governing insulin secretion, or by a hormonal mechanism modulating insulin sensitivity and acting through hypothalamus and pituitary. A hormonal component is implied by the observation that alterations in insulin sensitivity as well as secretion must be invoked to explain all the glucose and insulin patterns observed in paired $A M$ and PM tests $[13,14]$. Cortisol and growth hormone are the two most important insulin antagonists known to be under hypothalamic control; as plasma levels of the former are known to be highest in the morning, we examined changes in GH during the present series of tests.

The well recognised lability of basal GH values in young women $[15]$ is reflected in the very wide range of fasting $\mathrm{GH}$ values, and our findings provide no evidence that GH contributes significantly to diurnal variations in glucose tolerance. This contrasts with, for instance, the Somogyi effect, where an insulin antagonist effect of GH is probably important [16].

The observed relationship between obesity and diurnal glucose tolerance rhythm confirms the original observation of Jarrett and Keen [3]. Obesity is known to be associated with insulin resistance, and it is likely that subjects who become obese without developing diabetes are producing and secreting considerably more insulin than their counterparts of normal weight. This difference could be one factor contributing to the uniformity of glucose tolerance seen during the day in obese subjects. It has been suggested that obesity and oral contraceptive use may have many metabolic features in common [2]; however, the effects of obesity and oral contraceptives on diurnal glucose tolerance rhythm seem to be different. Thus when trying to assess the significance of impaired carbohydrate tolerance in patients on oral contraceptives, it must be 
recognised that the observed morning abnormalities are likely to be present to an exaggerated extent, in the afternoon. It is still uncertain whether this degree of chemical diabetes is harmful to the asymptomatic "pill" user.

Acknowledgements. This work was partly funded by a grant from the M.R.C. and we wish to acknowledge the receipt of grants from the British Diabetic Association (N.W.O.) and the Canadian Medical Research Council (D.M.).

\section{References}

1. Wynn, V., Doar, J.W.H. : Some effects of oral contraceptives on carbohydrate metabolism. Lancet 1969 II, $761-766$.

2. Doar, J.W.H., Wynn, V.: Effects of obesity, glucocorticoid and contraceptive therapy on plasma glucose and blood pyruvate levels. Brit. med. J. 1970 I, $149-$ 153.

3. Jarrett, R.J., Keen, H.: Further observations on the diurnal variation in oral glucose tolerance. Brit. med. J. 1970 VI, 334-337.

4. Jarrett, R.J., Keen, H.: Diurnal variation of oral glucose tolerance: a possible pointer to the evolution of diabetes mellitus. Brit. med. J. 1969 I, 341-345.

5. Cramp, D.G.: New automated method for measuring glucose by glucose oxidase. J. clin. Path. 20, 910-912 (1967).

6. Albano, J., Ekins, R.P., Maritz, G., Turner, R.C.: A sensitive precise radio-immunoassay of serum insulin relying on charcoal separation of bound and free hormone moieties. Acta endocr. 70, 487-509 (1972).

7. Jacobs, H.S.: Use of activated charcoal in the radioimmunoassay of human growth hormone in plasma. J. clin. Path. 22, 710-717 (1969).
8. Metrop. Life Insur. Co. Statist. Bull. 40 (1959).

9. Turner, R.C., Oakley, N.W., Nabarro, J.D.N.: Control of basal insulin secretion with special reference to the diagnosis of insulinomas. Brit, mod. J. $1971 \mathbf{I}$, $132-135$.

10. Welborn, T.A., Rubenstein, A.H., Haslam, R., Fraser, R.: Normal insulin response to glucose. Lancet 1966 I, $280-284$.

11. Roberts, H.J.: Afternoon glucose tolerance testing: a key to the pathogenesis, early diagnosis and prognosis of diabetogenic hyperinsulinism. J. Amer. Geriat. Soc. 12, $423-472(1964)$.

12. Bowen, A.J., Reeves, R.L.: Diurnal variation in glucose tolerance. Arch. int. Med. 119, 261-264 (1967).

13. Jarrett, R.J., Baker, I.A., Keen, H., Oakley, N.W. Diurnal variation in oral glucose tolerance: blood sugar and plasma insulin levels morning, afternoon and evening. Brit. med. J. 1972 I. $199-201$.

14. Gibson, T., Jarrett, R.J.: Diurnal variation in insulin sensitivity. Lancet 1972 II, 947-948.

15. Roth, J., Glick, S.M., Yalow, R.S., Berson, S.A.: Secretion of human growth hormone: physiologic and experimental modification. Metabolism 12, 577-579 (1963).

16. Oakley, N.W., Jacobs, H.S., Turner, R.C., Williams, J., Aquino, C dos S., Nabarro, J.D.N.: The effect of hypoglyca emia on oral glucose tolerance in normal subjects and patients with pituitary and adrenal disorders. Clin. Sci. 39, 663-674 (1970).

\section{Dr. N.W. Oakley}

Alexander Simpson Laboratory

for Metabolic Research

St. Mary's Hospital

Medical School

London W. 2

England 